\title{
Multiple facets of intestinal permeability and epithelial handling of dietary antigens
}

\author{
S Ménard ${ }^{1}, \mathrm{~N}_{\text {Cerf-Bensussan }}{ }^{1}$ and $M$ Heyman $^{1}$
}

The intestinal epithelium, the largest interface between the host and environment, regulates fluxes of ions and nutrients and limits host contact with the massive load of luminal antigens. Local protective and tolerogenic immune responses toward luminal content depend on antigen sampling by the gut epithelial layer. Whether, and how exaggerated, the entrance of antigenic macromolecules across the gut epithelium might initiate and/or perpetuate chronic inflammation as well as the respective contribution of paracellular and transcellular permeability remains a matter of debate. To this extent, experimental studies involving the in vivo assessment of intestinal permeability using small inert molecules do not necessarily correlate with the uptake of larger dietary antigens. This review analyzes both the structural and functional aspects of intestinal permeability with special emphasis on antigen handling in healthy and diseased states and consequences on local immune responses to food antigens.

\section{INTRODUCTION}

The intestinal epithelium forms a selective barrier, which favors fluxes of nutrients, regulates ion and water movements, and limits host contact with the massive intraluminal load of dietary antigens and microbes. However, this barrier is not fully impermeable to macromolecules; in the steady state, the transepithelial passage of small amounts of food-derived antigens and microorganisms participates in the induction of a homeostatic immune response dominated by immune tolerance to dietary antigens ${ }^{1,2}$ and the local production of secretory immunoglobulin A (SIgA), ${ }^{3}$ preventing pathogenic and commensal microbes from entering internal compartments. Conversely, primary or secondary defects of the intestinal barrier can lead to excessive entrance of dietary or microbe-derived macromolecules, which are putative contributors to the pathogenesis of a spectrum of human diseases, including food allergy and inflammatory bowel diseases (IBDs), and could even be related to autoimmune diseases and metabolic syndrome. ${ }^{4}$ Reinforcing the intestinal barrier and more particularly the paracellular pathway has recently been suggested as a therapeutic strategy to treat or prevent diseases driven by luminal antigens. Delineating how antigens are transported across the epithelium in healthy and diseased states should help in the design of appropriate therapeutic tools.
Herein, we will discuss the multiple pathways involved in the intestinal transport of luminal food antigens and analyze the contribution of the paracellular and transcellular pathways.

\section{DIETARY ANTIGENS ARE AVAILABLE FOR INTESTINAL TRANSPORT}

Although the majority of dietary proteins are totally degraded by digestive enzymes and are absorbed in the form of nutrients (amino acids or dipeptides/tripeptides), some however can resist both the low $\mathrm{pH}$ of the gastric fluid and proteolytic enzyme hydrolysis, ${ }^{5}$ meaning that large immunogenic peptides or intact proteins are capable of reaching the small intestinal lumen. ${ }^{6}$ For example, $\beta$-lactoglobulin, a major cow's milk allergen, is stable under acidic conditions and resists digestion by pepsin, whereas the resistance of gluten/gliadins to digestive enzymes is a major factor underlying celiac disease (CD). The high proline content (20\%) of gliadins prevents their efficient intraluminal digestion and leads to the release of large irreducible 33- and 26 -mer immunogenic peptides ${ }^{7,8}$ able to activate the lamina propria $\mathrm{CD} 4^{+} \mathrm{T}$ cells in celiac patients. The deleterious role of impaired protein digestion is highlighted by the increased risk of food allergy reported in patients taking antiulcer medication, which likely impairs gastric protein digestion. ${ }^{9}$ Despite this

${ }^{1}$ INSERM, U989, Interactions of the intestinal epithelium with the immune system, Université Paris Descartes, Paris, Cedex 15, France. Correspondence: M Heyman (martine.heyman@inserm.fr)

Received 15 December 2009; accepted 28 January 2010; published online 10 March 2010. doi:10.1038/mi.2010.5 
correlation between resistance to hydrolysis and allergenicity/ toxicity, a causal relationship is not always the rule as some food allergens are pepsin sensitive (shrimp tropomyosin, milk caseins, $\alpha$-lactalbumin, bovine serum albumin) (JM Wal, personal communication) and small fragments generated during digestion can bind IgE. ${ }^{10}$ Taken together, these observations indicate that immunogenic proteins and peptides can be present in the intestinal lumen and thus can serve as potential candidates for intestinal absorption and immune stimulation.

\section{INTESTINAL STRUCTURES CONTROLLING PARACELLULAR ANDTRANSCELLULAR PERMEABILITY Definition of intestinal permeability}

"Intestinal permeability," "intestinal permeation," and "intestinal transport" are terms often used indistinctly to describe the movement of molecules across the gut wall. However, intestinal permeability (expressed in $\mathrm{cm} \mathrm{s}^{-1}$ ) is an intrinsic property of the intestine and is defined as "the facility with which intestinal epithelium allows molecules to pass through by non-mediated passive diffusion;"11 this concept mainly refers to the passage of ions and inert molecules of low molecular weight. In contrast, intestinal transport is measured by unidirectional fluxes (expressed in $\mathrm{molh}^{-1} \mathrm{~cm}^{-2}$ ) and relates to the number of molecules crossing the epithelial layer in a given time. The intestinal transport of molecules from the intestinal lumen to the lamina propria can occur through two distinct mechanisms: paracellular diffusion through tight junctions (TJs) between adjacent intestinal epithelial cells (IECs) and transcellular transport involving endocytosis/exocytosis (transcytosis) mediated or not by membrane receptors.

\section{Structural components of the intestinal epithelium determining intestinal permeability}

The gut epithelial monolayer issues from a pool of pluripotent stem cells, which are located at the bottom of the crypts, ${ }^{12}$ and give rise to five types of epithelial cells: absorptive columnar cells (enterocytes), and goblet, endocrine, Paneth, and M (microfold) cells. Epithelial cohesion and polarity are maintained by the apical junctional complex, which is composed of tight and adherens junctions, and by the subjacent desmosomes (Figure 1). The most apical structures, the TJs, are the rate-limiting factor for the epithelial paracellular permeation of molecules. The other structures maintain cell proximity but are not rate limiting as the width of the lateral space below the TJ is estimated to be $75 \AA^{13}$ compared with 4-9 or $50-60 \AA$ for the TJ pores in villi or crypts, respectively. ${ }^{14-16} \mathrm{TJ}$ can be visualized in freeze-fracture electron microscopy images as strands and grooves, with a higher number of strands found in villous TJ than in crypt TJ. ${ }^{17}$ This suggests a decreasing gradient of paracellular permeability from crypt to villous IECs. A large set of structural and regulatory molecules controls the plasticity and permeability of TJ, ${ }^{18}$ which are mainly composed of proteins, including occludin, ${ }^{19}$ claudins, ${ }^{20}$ JAM-A (junctional adhesion molecule A), ${ }^{21}$ and tricellulin. ${ }^{22}$ Occludin, similar to claudins, interacts directly with membrane-associated zonula occludens (ZO)-1, ZO-2, and ZO-3 proteins that regulate the perijunctional actinomyosin ring.

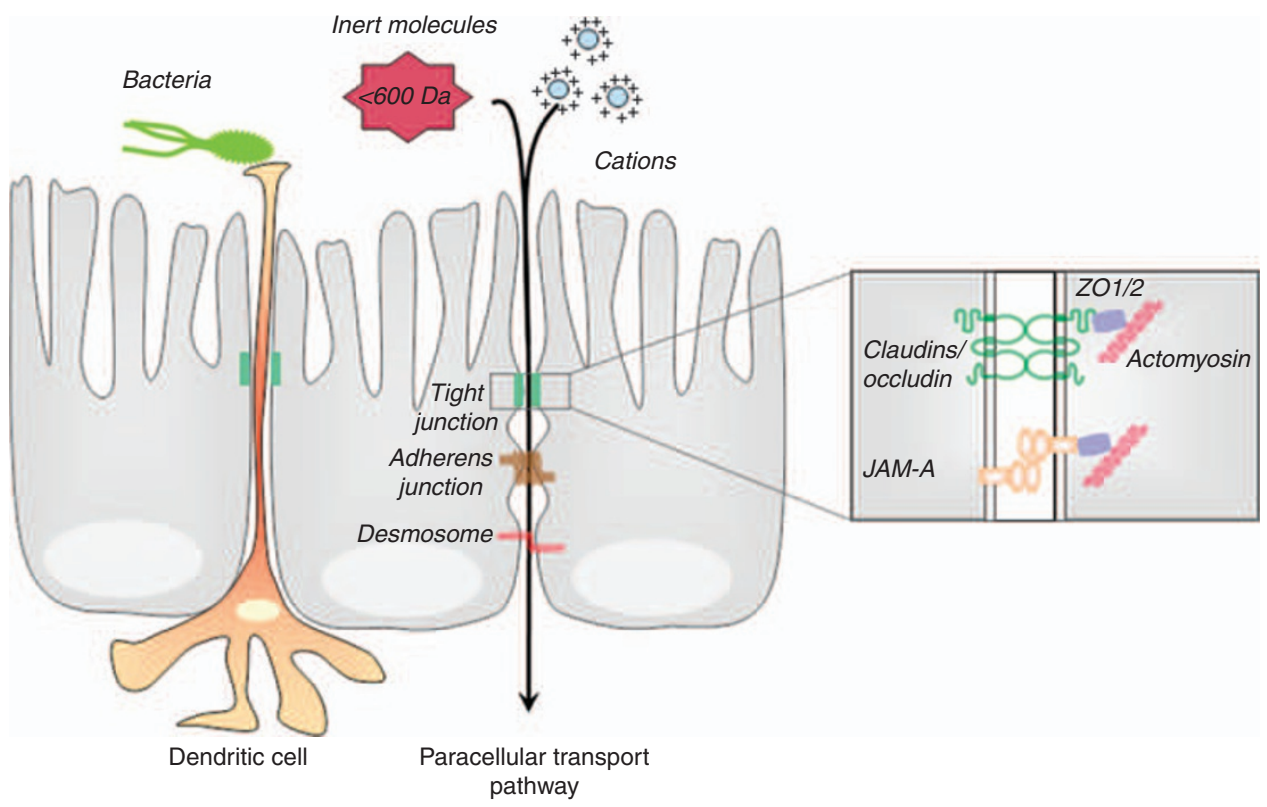

Figure 1 Paracellular transport pathway. The paracellular pathway relates to structures joining adjacent intestinal epithelial cells and is delineated by tight junctions, adherens junctions, and desmosomes. Paracellular diffusion of molecules is mainly restricted by tight junctions, a network of transmembrane proteins (claudins, occludin, junctional adhesion molecule A (JAM-A)) forming pores of $\sim 8 \AA$ diameter and connected to the actomyosin ring through zonula occludens proteins (ZO-1, ZO-2). Under steady-state condition, these highly regulated structures allow the diffusion of ions (mostly cations) and inert molecules of small size (molecular weight (MW) $<600 \mathrm{Da}$, mannitol, lactulose) often used as permeability markers. Paracellular permeability can be increased in various pathological situations in which molecules of higher MW can diffuse nonspecifically across the epithelial layer. 
Claudins, a large family consisting of 24 members, ${ }^{20,23,24}$ determine the charge selectivity of the paracellular pathway. ${ }^{25,26}$ In the intestine, claudin-2 forms cation-selective channels in TJ. ${ }^{27}$ A tightness function has been described in various epithelia for claudin-1, -3, -4, -5, -8, -11,-14, and -19 using knockout mouse models or a range of assays (cited in Krause et al. ${ }^{28}$ ). Other claudins such as claudin-2, $-7,-10,-15$, and -16 , are poreforming molecules likely to increase paracellular permeability as exemplified in claudin-15 knockout mice, for which ionic conductance is decreased compared with control littermates. ${ }^{29}$ The junctional protein JAM-A is also involved in the barrier function, and decreased JAM-A expression has been reported in IBDs. ${ }^{30}$ In $J A M$ - $A$-deficient mice, increased intestinal permeability (and decreased intestinal electrical resistance) is related to the upregulation of claudin-10 and claudin-15. ${ }^{31}$ Finally, tricellulin, an integral membrane protein contributing to the structure and function of tricellular contacts between neighboring cells, has a critical role in the formation of the epithelial barrier. ${ }^{22}$

Besides permeability to small molecules through the paracellular transport pathway, a transcellular transport pathway allows large antigenic molecules to gain access to the subepithelial compartment and to interact with local immune cells. Macromolecules can be sampled from the intestinal lumen into enterocytes by a vesicular transport (fluid phase or receptormediated endocytosis) and released basolaterally. Owing to their structural and molecular features, including receptor expression on apical surfaces (Toll-like receptors, lectin-like microbial adhesins, $\alpha 5 \beta 1$ integrin, platelet-activating factor receptor or glycoprotein-2), ${ }^{32,33} \mathrm{M}$ cells located in the follicle-associated epithelium of Peyer's patches (PPs) are particularly involved in the transcytosis of bacteria. In parallel, columnar enterocytes, the major cell type in the small intestinal epithelium, can sample, transport, and/or process soluble antigens all along the intestine. The important role of columnar enterocytes in the sampling of soluble antigens is highlighted by studies indicating (i) that antigen transport through afferent lymphatics into the draining mesenteric lymph nodes is obligatory for oral tolerance induction; ${ }^{34}$ (ii) that PPs are not mandatory for the development of oral tolerance; ${ }^{35}$ and (iii) very rapid antigen uptake by dendritic cells (DCs) in the small intestinal lamina propria after feeding mice with ovalbumin (OVA). ${ }^{36}$

\section{THE PARACELLULARTRANSPORT PATHWAY: A REGULATED FORM OF MOLECULAR SIEVING}

Paracellular permeability is related to the pores in the epithelial TJ determining a high-capacity, size-restricted pathway and a low-capacity, size-independent (or less restrictive) pathway that might be due to fixed (e.g., tricellular junctions, larger pores) or transient breaks (e.g., apoptosis) in the epithelial monolayer. ${ }^{37}$ The paracellular pathway accepts molecules with molecular mass $<600 \mathrm{Da}^{38}$ (Figure 1); permeation activity through this pathway can be measured from the diffusion of small inert probes (probe diameter: PEG400 $=5.3 \AA$, mannitol $=6.7 \AA$, rhamnose $=8.3 \AA$, lactulose $=9.5 \AA$, cellobiose $=10.5 \AA$, ${ }^{51} \mathrm{Cr}-$ EDTA $=11 \AA$ ). Paracellular permeability is mainly determined by pore size in TJ, with most pores being in the range $8-9 \AA$ in diameter. ${ }^{38}$ A pore theory relying upon calculation ${ }^{11,39}$ suggested that a large number of small pores together with a small number of large pores could explain the higher permeation of small-sized markers and vice versa. This theory fits with recent findings suggesting that both small and large pores are defined by different TJ proteins, such as claudins and tricellulin, respectively. The paracellular diffusion of small molecules through TJ pores is driven by water movement due to transepithelial electrochemical or osmotic gradients that induce solvent drag. A typical example is $\mathrm{Na}^{+}$-glucose cotransport that creates a $\mathrm{Na}^{+}$ gradient driving the mucosal to serosal flux of water and consequently of small solutes. ${ }^{40}$ The case of mannitol permeability illustrates that epithelial transport pathways depend on both structural features and driving forces. Mannitol is often used as a paracellular marker in vitro (epithelial cell monolayers or intestinal fragments in Ussing chambers), but is considered a marker of transcellular permeability in vivo when permeability is measured by the differential absorption of lactulose and mannitol in clinical studies. This discrepancy originates from the distinct driving forces moving mannitol in vivo and in vitro. In vivo, the hyperosmolality of villus tips due to blood flow creates a powerful solvent drag effect that is absent in vitro. Thus, whereas the intestinal permeability test (IPT) can discriminate between paracellular (lactulose) and transcellular (mannitol) pathways in vivo, in contrast, in vitro, the human intestine does not discriminate between mannitol- and lactulose-sized molecules. ${ }^{41}$

\section{INTESTINAL ELECTRICAL RESISTANCE AND LACTULOSE/ MANNITOL PERMEABILITY TEST: ARE THEY CORRELATED WITH ANTIGENTRANSPORT?}

Gold standards to measure intestinal permeability are tissue electrical resistance (in vitro) and lactulose/mannitol IPT (in vivo). Although electrical resistance provides an approximate measurement of the transjunctional flux of ions, ${ }^{42}$ inert sugars and food antigens obviously do not behave like ions. Electrical resistance and the permeation of inert sugars, two indicators of paracellular permeability, are not linearly correlated and their degree of correlation worsens as the molecular size of the solute increases. ${ }^{43}$ IPT is a useful test in clinical studies, giving information on the overall status of the digestive tract (villous atrophy, inflammation); however, this test is not indicative of the status of macromolecular transport. Indeed, studies have shown the lack of correlation between the permeation of inert sugars and macromolecules. In neonatal guinea pigs, intestinal closure to $\beta$-lactoglobulin (molecular weight $18,000 \mathrm{Da}$ ), a major allergen in cow's milk, occurs within 6 days of birth, whereas permeation of lactulose, a marker of paracellular permeability, persists throughout the suckling period. This indicates that inert soluble markers do not trace macromolecular absorption and do not reflect antigen handling by the gut. ${ }^{44}$ Another example is provided in children with rotavirus diarrhea, wherein lactulose/mannitol IPT and the absorption of $\beta$-lactoglobulin are not directly correlated. ${ }^{45}$ Finally, in a mouse model of celiac-like disease (HLA-DQ8-HCD4), mice challenged with 
gluten present increased fluxes of horseradish peroxidase (HRP, a macromolecular tracer) in the absence of increased ionic conductance, whereas the addition of indomethacin to gluten promotes an increase in ionic conductance (paracellular pathway) and a further increase in HRP transcytosis. Thus, one should keep in mind that electrical resistance (or its reverse ionic conductance) is mainly related to the permeation of ions, and at best, of small molecules, but not always to the permeation of food-type antigens. Another important issue to consider is the measurement of intestinal permeability in experimental animals in vivo, by testing the presence of macromolecular tracers (dextrans or Evans Blue) in the blood after gavage. These techniques should not be considered as state-of-the-art methods to measure intestinal permeability, as they do not take into account important factors such as gastrointestinal motility (affecting the time of contact of the tracer with the mucosa) and body distribution of the tracers, which can significantly affect the measurement of intestinal permeability. Although more difficult to handle, intestinal loop systems and tracer recovery in mesenteric or portal blood ${ }^{46,47}$ should be considered in animal models as a gold standard to measure intestinal permeability in vivo.

\section{TRANSCELLULARTRANSPORT PATHWAYS}

The transcellular transport of large particles (Figure 2), including microbes, has been traditionally ascribed to $\mathrm{M}$ cells overlying PPs and isolated lymphoid follicles in the distal part of the mice $^{48}$ and human intestine. ${ }^{49,50}$ Alternatively, intestinal DCs have the capacity to sample bacteria directly in the intestinal lumen by extending dendrites between epithelial cells. ${ }^{51}$ The role of $\mathrm{M}$ cells in the sampling of soluble antigens is not exclusive, and food antigens present in the proximal intestine can be transported through columnar enterocytes. This transcytosis mechanism was initially described in rats in the 1970 s. $^{52}$ It was subsequently explored in other animal models ${ }^{53,54}$ and validated in the human intestine. ${ }^{55}$ As an example, $\beta$-lactoglobulin was shown by immunohistochemistry to enter epithelial cells in organ cultures of human duodenum. ${ }^{56}$ In ex vivo studies of animal and human intestinal mucosa, the uptake of food antigens by IEC was associated with a powerful epithelial degradation. ${ }^{57}$ Using biopsies mounted in Ussing chambers with tritiated food proteins ( ${ }^{3} \mathrm{H}$-lysines) added to the mucosal compartment, transport and processing/degradation during transcytosis could be quantified by analysis of ${ }^{3} \mathrm{H}$-labeled fragments released in the serosal compartment. ${ }^{57}$ These studies indicated that only small amounts of intact protein are transcytosed $(\sim 0.1 \%$ of luminal concentration), illustrating the efficient barrier function of the gut epithelium. The latter experiments also indicated that epithelial cells process proteins into peptides of $\sim 1,500 \mathrm{Da}$ molecular weight, a size compatible with binding into the peptide pocket of antigen-presenting major histocompatibility complex (MHC) class II molecules. ${ }^{58}$ Quantification by high-performance liquid radiochromatography indicated that large proteins taken up by IECs were released on their basal pole either as immunogenic peptides $(\sim 40 \%)$ or fully degraded into amino acids ( $50 \%)$, with only a minor fraction crossing the epithelium in their intact form $(<10 \%) .{ }^{53}$ Large peptides or proteins released into

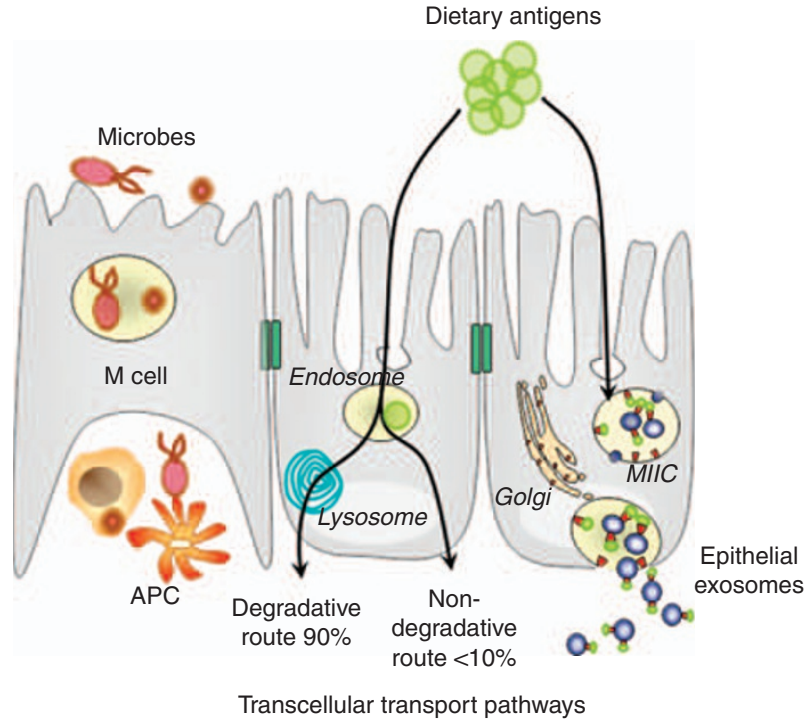

Figure 2 Transcellular transport pathways. Under steady-state condition, molecules of molecular weight (MW) $>600 \mathrm{Da}$ (such as food antigens, peptides) are sampled by the epithelial cells by endocytosis at the apical membrane and transcytosis toward the lamina propria. During transcytosis, full-length peptides or proteins are partly degraded in acidic and lysosomal compartments and released in the form of amino acids (total degradation) or breakdown products (partial degradation) at the basolateral pole of enterocytes. Early endosomes containing partially degraded food antigens meet the major histocompatibility complex (MHC) class II-enriched compartment (MIIC) where exogenous peptides are loaded on MHC class II molecules. Inward invagination of MIIC compartment lead to the formation of exosomes, which are small membrane vesicles $(40-90 \mathrm{~nm}$ ) bearing MHC class II/peptide complexes at their surface. Exosomes can diffuse in the basement membrane and interact with local immune cells. Exosome-bound peptides are much more potent than free peptides to interact with dendritic cells and stimulate peptide presentation to $T$ cells.

the lamina propria might then be taken up by local antigen-presenting cells, as demonstrated in the study by Chirdo et al. ${ }^{36}$ who showed that, after gavage of mice with OVA, antigen was rapidly associated with small intestinal lamina propria DC. The fact that a consistent fraction of transcytosed food antigens presents as small immunogenic peptides suggested that protection from total degradation might occur during transcytosis. The notion that antigen-presenting cells can process proteins and release vesicles bearing protein-derived peptides bound to MHC class II molecules $^{59}$ (exosomes) led us to examine whether IECs might release immunogenic peptides loaded on exosomes.

\section{The role of exosomes in the transport of food-derived immunogens}

This hypothesis was supported by our demonstration that IECs produce vesicular structures (Figure 2), with structural and molecular features similar to those of antigen-presenting vesicles known as exosomes produced by immune cells. ${ }^{59}$ Exosomes are small membrane vesicles ( $~ 80 \mathrm{~nm}$ in diameter) first described in reticulocytes and hematopoietic cells. ${ }^{60}$ In professional antigenpresenting cells, exogenous antigens are endocytosed at the cell surface and are processed in early endosomes before reaching 
MHC class II-enriched compartments (MIICs) where antigenderived peptides are loaded on MHC class II molecules. In this compartment, exosomes are formed by inward invagination of the MIIC-limiting membrane, explaining why they carry MHC class II/peptide complexes at their surface. MIICs can either be directed to the lysosomal compartment or fuse with the plasma membrane and release exosomes into the extracellular medium. Similarly, the capacity of epithelial cells to release exosome-like vesicles bearing HLA-DR/peptide complexes ${ }^{61}$ was identified as a mechanism whereby luminal antigens are transferred in a highly immunogenic form to the lamina propria (Figure 2). Thus, in vitro studies have shown that peptides derived from epithelial processing and released bound to exosomes interacted very efficiently with DCs and could stimulate antigen-specific T-cell clones at concentration 100-fold less than free peptides. ${ }^{62}$ Furthermore, systemic in vivo administration of epithelial exosomes primed a potent immunogenic response. ${ }^{63}$ Therefore, epithelium-derived exosomes may be potent vehicles involved in intestinal antigen presentation. The in vivo outcome of immune stimulation by epithelium-derived exosomes likely depends on the nature of the lamina propria antigen-presenting cells, and notably on the conditioning of DC by epithelium-derived factors. ${ }^{64,65}$ Interestingly, in vivo studies supported the tolerogenic effect of epithelium-derived exosomes. ${ }^{66,67}$ It is therefore tempting to speculate that their uptake in the small intestinal lamina propria by tolerogenic $\mathrm{CD} 103^{+} \mathrm{DC}$ may promote oral tolerance. The transcytosis of food antigens commences primarily by a fluid-phase endocytosis of proteins at the apical membrane of enterocytes; however, under different circumstances, pathogenic antigens can access the mucosa through the expression of Ig receptors at the apical surface of enterocytes, thereby allowing their entry in the form of immune complexes (ICs).

\section{Antigen transcytosis through ICs (IgA, IgE, IgG)}

IgA-mediated transport. IgA is the most representative Ig isotype at the mucosal surface. A common receptor-mediated IgA transport mechanism in the intestine is the basal-to-apical secretion of dimeric IgA in the form of SIgA through the polymeric Ig receptor. SIgA constitutes an important aspect of intestinal protective immunity by retaining potentially noxious antigens ${ }^{68,69}$ in the intestinal lumen. Although SIgAs are mainly devoted to restricting the entry of exogenous antigens in the intestinal mucosa, some cases of apical-to-basal retrotransport have been reported, with either beneficial or deleterious effect on the intestinal mucosa.

In rodent PPs, an unknown IgA receptor was shown to mediate the apical-to-basal transcytosis of $\operatorname{Ig} \mathrm{A}^{70,71}$ or $\operatorname{IgA}$ bound to viruses or bacteria ${ }^{72,73}$ across $\mathrm{M}$ cells. In animal models, administration of Shigella flexneri alone or as SIgA ICs into ligated intestinal loops containing PPs, allowed IC, but not free bacteria, to enter PP and to be captured by DC (Figure 3), thereby contributing to the induction of protective immunity ${ }^{72}$ and preserving intestinal barrier integrity. ${ }^{74}$

Whereas retrotransport of SIgA/bacteria IC aids in the development of immune responses to clear pathogenic microbes, this retrotransport might turn deleterious to the host when food antigens are concerned. This is the case in CD, an enteropathy induced by the abnormal activation of $\mathrm{T}$ cells by gluten-derived gliadin peptides. In CD, gliadin peptides are transported intact across the intestine through IgA/gliadin $\mathrm{IC}^{75}$ (Figure 3). This abnormal "protected" transport of gliadin is due to the ectopic expression of the transferrin receptor CD71, known to bind IgA $1,{ }^{76}$ at the apical surface of IECs. Indeed, whereas in healthy individuals, CD71 is exclusively expressed at the basolateral membrane of IEC, its apical expression in active CD patients allows the retrotransport of SIgA/gliadin IC in the lamina propria. In contrast, gliadin peptides are almost totally degraded (detoxified) by IECs during intestinal transport in healthy individuals, wherein epithelial CD71 is expressed basolaterally and no SIgA IC retrotransport is observed. Although a clear relationship between SIgA/gliadin IC in the lamina propria and pathogenic outcome in celiac patients has not yet been established, it is highly probable that IgA-mediated gliadin transport is involved in the overstimulation of the local immune system. Thus, although the IgA-mediated retrotransport of pathogenic bacteria might be beneficial to improve bacterial clearance and restoration of intestinal homeostasis, as discussed above, ${ }^{70,72}$ the same mechanism applied to a normally nonpathogenic antigen such as gliadin might be deleterious rather than protective, in view of the constant flow of gluten in the gut. In addition, the presence of large aggregates of gliadin-specific IgA in duodenal secretions, lamina propria, and serum of celiac patients could provide a danger signal promoting the rupture of oral tolerance and/or triggering tissue damage. The damaging effects of large IgA complexes deposited in tissues have been exemplified in IgA nephropathy. ${ }^{77}$

IgE-mediated transport. The human low-affinity receptor for IgE (FceRII, CD23) ${ }^{78}$ can mediate the transport of IgE IC in food allergy (Figure 4). CD23 is mainly expressed on hematopoietic cells, but it is also observed on the apical and basal surfaces of IECs in patients with gastrointestinal diseases that depend or not on IgE (IgE-dependent cow's milk allergy, autoimmune enteropathy, cow's milk protein enteropathy, Crohn's disease (CrD), and ulcerative colitis (UC)). ${ }^{79}$ High levels of interleukin (IL)-4, a Th2 cytokine involved in allergic diseases, enhances the expression of CD23. Although IgE is not considered as a secreted Ig, it can be found in lavage fluids in parasitic infection ${ }^{80}$ or in food allergy. ${ }^{81}$ The role of epithelial CD23 and IgE IC in the mucosal entry of food allergens has been unraveled in rodent models of allergy. Sensitization of rats to HRP led to increased HRP uptake into IECs and faster transcellular transport compared with naive control rats. ${ }^{82}$ This enhanced transport, observed for sensitizing protein only, was shown to involve an IgEdependent receptor-mediated process. ${ }^{83}$ Indeed, immune sensitization enhanced CD23 expression on IECs and allowed allergens complexed with IgE to bypass epithelial lysosomal degradation, resulting in the entry of a large amount of intact allergens into the mucosa. ${ }^{84} \mathrm{CD} 23$ is expressed as two spliced forms, a and $b,{ }^{85}$ according to cell type and animal species. In mice, IECs express two CD23b alternatively spliced forms 


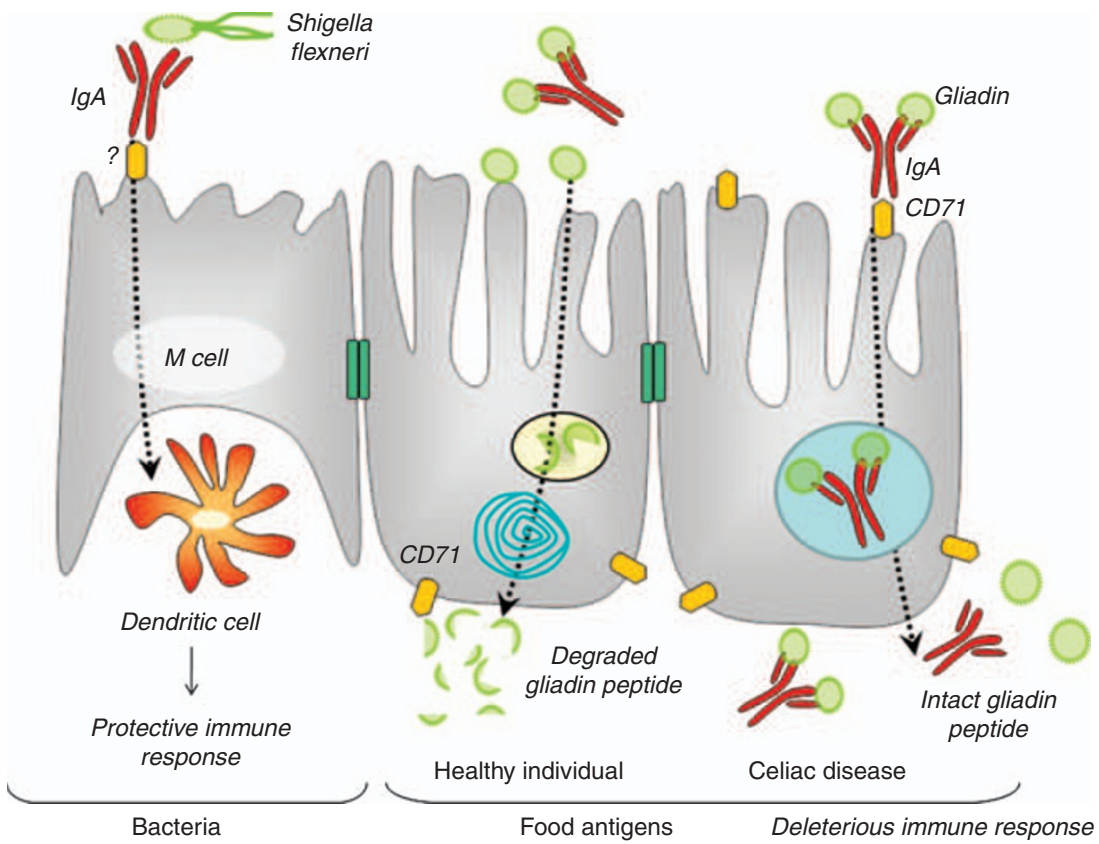

Figure $3 \mathrm{Immunoglobulin} \mathrm{(Ig)A-mediated} \mathrm{retrotransport} \mathrm{of} \mathrm{luminal} \mathrm{antigens.} \mathrm{IgA} \mathrm{is} \mathrm{a} \mathrm{protective} \mathrm{mucosal} \mathrm{immunoglobulin} \mathrm{secreted} \mathrm{in} \mathrm{the} \mathrm{intestinal}$ lumen through polymeric $\operatorname{lgR}(\mathrm{plgR})$ in the form of secretory $\lg \mathrm{A}(\mathrm{S} \lg \mathrm{A})$. Whereas the major role of SIgA is to contain microbial and food antigens in the intestinal lumen, in some pathological situations, an abnormal retrotransport of SIgA immune complexes can allow bacterial or food antigens entry in the intestinal mucosa, with various outcomes. Indeed, SIgA can mediate the intestinal entry of SIgA/Shigella flexneri immune complexes through $\mathrm{M}$ cells and interact with dendritic cells, inducing an inflammatory response aimed at improving bacterial clearance and the restoration of intestinal homeostasis. In celiac disease, however, SIgA allows the protected transcytosis of gliadin peptides, a mechanism more likely to trigger exacerbated adaptive and innate immune responses in view of the constant flow of gluten in the gut and to precipitate mucosal lesions. Indeed, whereas in healthy individuals, undigested gliadin peptides are taken up by nonspecific endocytosis in enterocytes and entirely degraded/detoxified during transepithelial transport, in contrast, in active celiac disease, the ectopic expression of CD71 (the transferrin receptor also known as IgA receptor) at the apical membrane of epithelial cells, favors the retrotransport of IgA immune complexes and inappropriate immune responses.

involved in the apical-to-basal transport of IgE or IgE IC, 86 whereas in human IEC lines, CD23b can transcytose IgE in a bidirectional manner. ${ }^{87}$ However, CD23a rather than CD23b is expressed in primary human IECs, and in transfected T84 IEC, CD23a acts as a bidirectional transporter of IgE and IgE IC. ${ }^{88}$ Thus, despite contradictory results on the involvement of CD23 a or b isoforms, it is likely that IgE IC delivered to the lamina propria after epithelial transport can degranulate mast cells, underlining the ability of the IC to activate local immune cells. This mechanism could participate in the rapid onset of intestinal symptoms in IgE-dependent food allergy.

IgG-mediated transport. Although IgA constitutes the predominant type of Ig at mucosal surfaces, gastrointestinal secretions contain significant amounts of IgG. It is now accepted that this feature is not related to the nonspecific diffusion of IgG through $\mathrm{TJ}$ as often suggested, but to a specific mechanism. Indeed, a bidirectional transport of IgG (Figure 4) occurs through the neonatal Fc receptor (FcRn), an MHC class I-related molecule composed of a heavy $\alpha$-chain in a noncovalent association with $\beta$-2-microglobulin. ${ }^{89,90}$ IgGmediated intestinal transport seems mostly implicated in protective immunity. The role of intestinal FcRn was initially reported in suckling rats that receive passive immunity from their mother by the intestinal absorption of IgG from the maternal milk. ${ }^{91,92}$ Such polarized absorption of IgG is explained by the binding properties of IgG to $\mathrm{FcRn}$ at an acidic $\mathrm{pH}(<6.5)$ recorded close to the apical membrane of IEC..$^{93-95}$ The dissociation of IgG from FcRn at neutral $\mathrm{pH}$ leads to $\mathrm{IgG}$ release on the basolateral side of the epithelia. In contrast, the human neonatal intestine is not a major site for the transfer of passive immunity, but $\mathrm{FcRn}$ can be found at the apical pole of enterocytes in fetal and adult intestine, ${ }^{96,97}$ even though the relevance of such expression has not been clearly established. Fc ligand valency influences the intracellular processing of IgG during transcytosis (protection vs. degradation); the Fc fragment displays two binding sites for $\mathrm{FcRn}$, and the presence of both binding sites is required for efficient transcytosis and protection of IgG from catabolism. ${ }^{98,99}$ The functional role of FcRn in the transfer of IgG IC has been characterized using polarized epithelial cell lines and transgenic mice. Polarized MDCK (Madin-Darby canine kidney) cells transfected with hFcRn were able to protect OVA from degradation during apical-to-basal transport of IgG/OVA IC, and OVA-specific $\mathrm{CD}^{+} \mathrm{T}$ cells were activated after transport of the IC. ${ }^{100}$ Moreover, in vivo studies on transgenic mice expressing $\mathrm{hFcRn}$ and $\beta_{2}$ microglobulin showed the FcRn-mediated transcytosis of IgG IC and their efficient presentation by $\mathrm{CD} 11 \mathrm{c}^{+} \mathrm{DC}$ to 


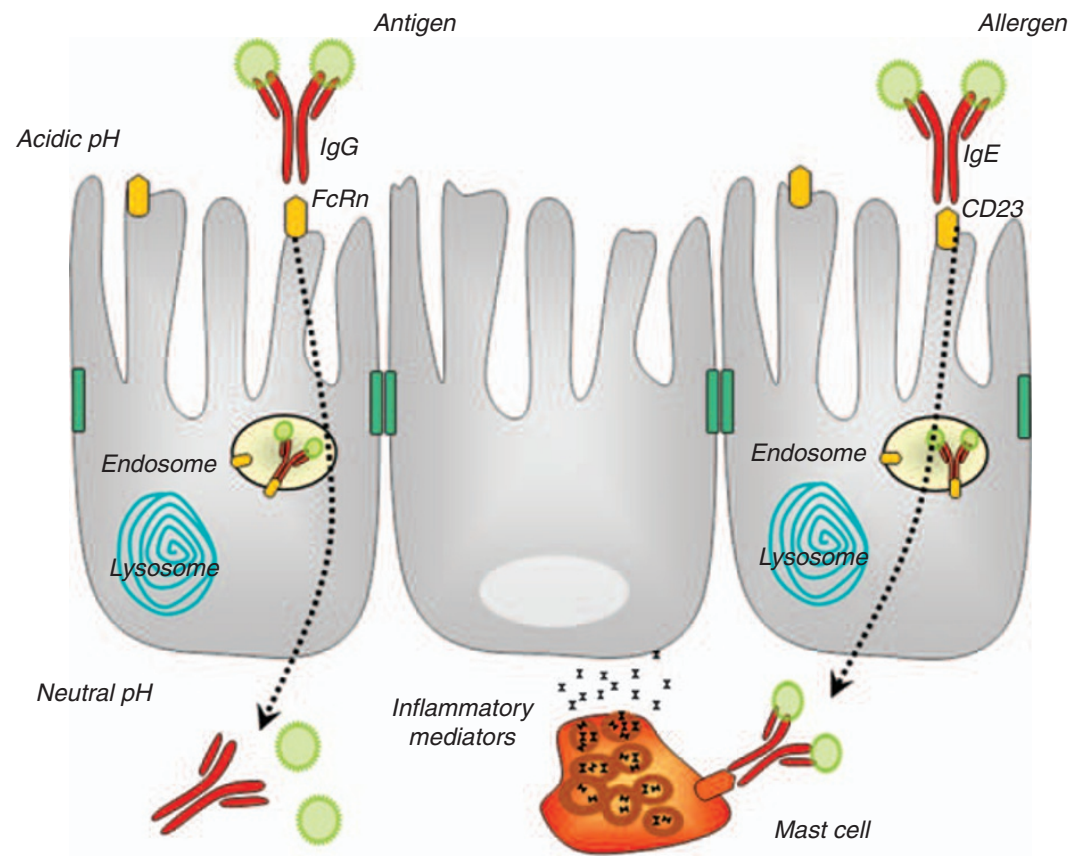

Figure 4 Immunoglobulin (Ig)G-mediated transport of antigens. Although IgGs are not classical secretory antibodies, their presence in the intestinal lumen suggests a protective role. IgGs have initially been shown to bind the neonatal Fc receptor on intestinal epithelial cells (FcRn) in the acidic environment close to the apical membrane or in early endosomes of enterocytes. This receptor-mediated transcytosis allows a protected transport of $\mathrm{IgG}$ and their release on the basal side of enterocytes where neutral $\mathrm{pH}$ induces their dissociation from the receptor. In vitro studies have indicated that IgG immune complexes can also be shuttled from the apical to the basal pole of enterocytes through FcRn and vice versa, although the incidence of IgG immune complexes in terms of immune response is not clearly established. IgE-mediated allergen transport. In food allergy, the low-affinity receptor for $\lg \mathrm{E}, \mathrm{CD} 23$, is abnormally overexpressed in intestinal epithelial cells, in humans and murine models of allergy. An overexpression of CD23 at the apical side of enterocytes can drive the transport of intact IgE/allergen immune complexes from the intestinal lumen to the lamina propria, a phenomenon triggering mast cell degranulation and allergic inflammatory cascade.

OVA-specific CD4 ${ }^{+}$T lymphocytes. ${ }^{100}$ Although the outcome of this immune response in vivo is not known, it has been reported that IgG IC might induce immune suppression. ${ }^{101,102}$ Not only food antigens but also bacteria can be transported as IgG IC through FcRn, a feature likely to have a role in the defense against intestinal pathogens. In this respect, recently reported data showed an increased sensitivity to Citrobacter rodentium in the absence of epithelial FcRn expression. Indeed, IEC-associated FcRn could retrieve IgG/C. rodentium IC from the intestinal lumen into the lamina propria where DC could take up the IC and subsequently activate $\mathrm{CD} 4^{+} \mathrm{T}$ cells in the mesenteric lymph nodes. ${ }^{103}$ This is likely to be a protective mechanism as $\mathrm{CD} 4^{+} \mathrm{T}$ cells have been shown to participate in the prevention of $C$. rodentium infection. ${ }^{104,105}$ Such a process has also been reported for commensal Escherichia coli, ${ }^{103}$ thus underlining a potential role of FcRn in the maintenance of intestinal immune homeostasis. In summary, permeation of IgA, IgE, or IgG IC in the intestinal mucosa depends mainly on the nature and the polarized expression of epithelial Ig receptors. ICs are likely to enhance local immune responses, with protective or deleterious immune responses according to the antigenic trigger. Indeed, mucosal entry of bacteria through IgG IC seems to aid in their immune clearance, but the entry of IC containing bacteria or bacterial by-products ${ }^{106}$ might also occasionally lead to chronic inflammation (IBD). In the case of food antigens, ICs seem to mostly induce deleterious immune responses, as exemplified in $\mathrm{CD}$ (IgA) and food allergy (IgE).

\section{TRANSPORT OF DIETARY ANTIGENS IN DISEASES: PARACELLULAR ORTRANSCELLULAR?}

It is generally accepted that luminal antigens can access the underlying immune system in digestive diseases more easily. However, it is hard to know whether large molecules are able to freely diffuse along disrupted epithelial junctions under inflammatory conditions or whether more subtle events, such as an increased rate of transcytosis, can drive the antigen flow toward the lamina propria. Moreover, whereas some digestive diseases are clearly related to dietary antigens (food allergy, CD), others such as IBD are linked more so to the abnormal entry of bacterial antigens.

\section{Paracellular permeability in diseases}

The release of proinflammatory cytokines, a hallmark of IBD, ${ }^{107-109}$ is responsible for increased intestinal permeability along both paracellular and transcellular pathways. The mechanisms underlying the structural and functional modifications of $\mathrm{TJ}^{110,111}$ include the endocytosis of junctional proteins, ${ }^{112,113}$ epithelial apoptosis or ulceration, ${ }^{114-116}$ the reduced transcription of TJ proteins, ${ }^{117}$ and the activation of myosin light chain kinase (MLCK) phosphorylation to promote cytoskeletal contraction. ${ }^{118}$ Indeed, MLCK is a key molecule stimulating the opening of TJ by 
phosphorylating MLC. ${ }^{119}$ Transgenic mice overexpressing MLCK exhibit increased protein transport (although it was not determined whether this phenomenon involved paracellular leakage or activated transcytosis) ${ }^{120}$ and although healthy, are much more prone to develop colitis. IL1- $\beta$ also enhances TJ permeability by stimulating mRNA transcription and activity of MLCK, ${ }^{121}$ suggesting that MLCK could be the target of diverse cytokines to regulate intestinal permeability. ${ }^{122-125}$

Among inflammatory cytokines, interferon (IFN) $\gamma$ was the first described to decrease transepithelial electrical resistance (TER) in the intestinal epithelial cell line T84 ${ }^{126}$ by inducing the migration of JAM-A, occludin, and claudin- 1 and -4 from the TJ domain to the apical membrane. ${ }^{127,128}$ These modifications of TJ protein distribution were shown to depend on a macropinocytosis-like process $^{128}$ and Rho/ROCK signaling. ${ }^{12}$ Tumor necrosis factor (TNF) $\alpha$ is also a major cytokine involved in IBD, and is known to decrease the thickness of $\mathrm{T}$ J strands and grooves network and to decrease TER. ${ }^{129,130}$ A synergistic effect of IFN $\gamma$ and TNF $\alpha$ in inducing damage to the epithelial barrier has been observed ${ }^{131}$ and could be due to both the IFN $\gamma$-induced expression of TNFR $2^{132}$ and the synergistic effect on myosin II light chain kinase (MLCK) activation. ${ }^{133}$ An alteration of TJ permeability induced by IFN $\gamma$ or TNF $\propto$ is also associated with an increased transcytosis of macromolecules (see below). IL-13, a cytokine overexpressed in UC, contributes to the low electrical resistance observed in colonic biopsies. ${ }^{134}$ In HT-29/B6 intestinal cell monolayers, IL-13 decreases TER by inducing apoptosis ${ }^{135}$ and by stimulating the expression of the pore-forming TJ molecule claudin-2. ${ }^{136}$ In UC, the presence of subapical vesicles containing TJ proteins suggests that the endocytosis pathway is involved in the modulation of TJ properties. ${ }^{11}$ An upregulated apoptotic rate within the colonic epithelium also contributes to the barrier defect ${ }^{130}$ in UC. IL-17, a recently identified cytokine having a role in IBD, was shown to strengthen the epithelial barrier ${ }^{137}$ in the T84 epithelial cell line, although the role of IL-17 in the formation and strengthening of $\mathrm{TJ}$ remains to be more fully studied.

Besides IBD, other environmental factors or diseases can influence paracellular permeability. Psychological stress is known to exacerbate symptoms of IBD and to decrease mucosal barrier function. ${ }^{138,139}$ Acute stress in rodents increases epithelial ionic conductance and permeation of small inert molecules (mannitol, ${ }^{51} \mathrm{Cr}$-EDTA) along the paracellular pathway; ${ }^{138,140}$ this stress-induced epithelial barrier defect also extends to the transcytosis of macromolecules with antigenic potential, ${ }^{141}$ underlining the fact that both paracellular and transcellular permeability are enhanced by stress. Part of the effect of stress on intestinal permeability probably derives from the release of neuroendocrine factors such as corticotropinreleasing hormone ${ }^{142}$ in the intestinal mucosa and the stimulation of cholinergic nerves. ${ }^{143}$ In contrast, the enteric nervous system can also control the tightness of the epithelial barrier as shown in a coculture model of epithelial cell lines and human submucosa in which the neuronal network reduces paracellular permeability and increases $\mathrm{ZO}-1$ expression through the release of vasoactive intestinal peptide. ${ }^{144}$ These later examples suggest that balanced positive and negative signals to the TJ participate in the regulation of paracellular permeability.
The role of paracellular permeability is also evident in CD in which a defect in the intestinal barrier capacity of celiac patients has been discussed as a potential trigger to the abnormal activation of the local innate and adaptive immune system. Since the discovery of ZO toxin, a vibrio cholerae toxin that disassembles intestinal TJ, a mammalian analog known as zonulin, recently identified as pre-haptoglobin-2, ${ }^{145}$ was reported; this factor might be responsible for modulating the paracellular leakage of gliadin in active $\mathrm{CD} .{ }^{146}$ The active fragment of zonulin increases intestinal permeability to mannitol ${ }^{147}$ and decreases transepithelial electrical resistance in epithelial cell lines and intestinal biopsies, ${ }^{148,149}$ although no direct evidence that zonulin increases the paracellular leakage of gliadin peptides has been provided to date. In addition, evidence that gliadin peptides cross the epithelial layer by transcytosis has recently been provided. ${ }^{150}$

\section{Transcellular permeability in diseases}

In $\mathrm{CrD}$, macromolecules can permeate the intestine at an increased rate. ${ }^{151}$ The molecular leak induced by TJ dysfunction is often associated with an increased transcytosis of luminal material. ${ }^{152}$ It has not been ruled out that paracellular permeation and transcytosis are connected together as both processes imply the remodeling of the cytoskeletal network. An increased epithelial uptake of OVA or HRP in colonic explants from $\mathrm{CrD}$ or UC patients compared with controls was observed by immune-electron microscopy. These proteins were taken up at the apical plasma membrane of enterocytes and reached the paracellular space by vesicular transport. ${ }^{153}$ This observation underlines the fact that visualization of macromolecules within the epithelial paracellular space does not mean that they have actually diffused through the TJ. Later studies indicated that the increased endosomal uptake of antigens was mediated by $\mathrm{TNF} \alpha .{ }^{152}$ This proinflammatory cytokine is not only involved in increasing TJ permeability but it also enhances transcellular transport. Consistent with this view, Söderholm et al., ${ }^{151}$ by comparing intestinal permeability with ${ }^{51} \mathrm{Cr}$-EDTA and OVA in active or inactive $\mathrm{CrD}$ patients, propounded that increased intestinal permeability observed in a subgroup of patients with inactive disease but at high risk of relapse, may be due to increased transcellular permeability occurring in the presence of an intact paracellular barrier and allowing the passage of antigenic molecules promoting inflammation. In line with this view, transcytosis of macromolecules is also enhanced by IFN $\gamma,{ }^{58}$ a Th 1 cytokine abundant in CrD. Finally, in IBD, a special type of epithelial cell known as RACE (rapid antigen uptake into the cytosol enterocyte) has been described, which is prone to transcytose antigens; ${ }^{154}$ in the mucosa of freshly resected biopsies from IBD and control specimens, OVA and HRP were transported to late endosomes and trans-Golgi vesicles of enterocytes. Therefore, in the inflamed gut, both paracellular and transcellular transport pathways are increased and contribute to the overstimulation of the local immune system. This creates a vicious circle in which luminal antigens reach the lamina propria, interact with immune cells, and drive the secretion of permeability-enhancing factors that contribute to further weaken the epithelial barrier function. 


\section{PERMEABILITY: CAUSE OR CONSEQUENCE OF INTESTINAL DISORDERS?}

A constant sampling of food antigens by IEC is mandatory for the development of oral tolerance. ${ }^{2}$ In this manner, sampled antigens that are immediately taken up by DCs in the lamina propria induce hyporesponsiveness to subsequent challenges. ${ }^{36}$ Whether a primary defect of the intestinal barrier might initiate digestive diseases and promote a shift from tolerance to sensitization due to the excessive absorption of antigens, is a longstanding hypothesis relevant to various digestive diseases, such as food allergy, CD, and IBD. However, constitutive abnormality in intestinal permeability is not recorded in food allergy. Indeed, in duodenal biopsies from infants with active cow's milk allergy, a significant increase in HRP transport was observed which, after treatment with cow's milk-free diet, returned to normal values. ${ }^{55}$ However, environmental factors that affect intestinal permeability, such as infection or stress, have consequences on susceptibility to allergic diseases (reviewed in Heyman ${ }^{155}$ ).

The increased intestinal permeability is an early biological change that often precedes the onset of autoimmune diseases, such as CD or type I diabetes. Such increased permeability could be due to environmental factors (such as infection, toxic molecules) that possibly initiate the disease. In CD, infection of the digestive tract might be a triggering factor that increases intestinal permeability to gliadin peptides and immune responses in susceptible individuals. Rotavirus infection has been reported as a possible candidate in the development of CD in children. Indeed, frequent rotavirus infection is associated with a higher risk of CD in early childhood. ${ }^{156}$

The increased permeability to intact gliadin peptides observed in active $\mathrm{CD}^{157}$ is not observed in most celiac patients treated with a gluten-free diet, even though a persistent increase in intestinal dysfunction (including permeability) is observed in some patients with treated CD. Thus, a primary defect in intestinal permeability in CD cannot be excluded, but this residual dysfunction is more likely related to the difficulty of patients to adhere to a strict gluten-free diet and/or to the persistent in situ increase in IFN $\gamma$ production.

The CD71-mediated transport of IgA/gliadin ICs ${ }^{75}$ occurring in active $\mathrm{CD}$ is linked to the overexpression of the transferrin receptor CD71. ${ }^{75}$ Although intestinal overexpression of CD71 is observed in $\mathrm{CD},{ }^{75,158}$ it is also observed in iron deficiency anemia. Notably, CD has been related to pregnancy ${ }^{159}$ and is known to affect females more than males. ${ }^{160}$ Therefore, it is not excluded that iron deficiency anemia might be involved in the initiation of disease, by promoting the abnormal delivery of gliadin in the intestinal mucosa through IgA receptors (i.e., transferrin receptor CD71), in susceptible individuals. Another condition possibly influencing CD71 expression is the proliferation status of IEC. A high proliferation rate secondary to epithelial damage is found in rotavirus infection ${ }^{161}$ and might also stimulate CD71 overexpression, thereby favoring the development of CD.

In IBD, the hypothesis of a primary defect of the intestinal barrier stems from observations of increased permeability in CrD patients up to 1 year before clinical relapse, and even more strikingly of an increased IPT result in healthy relatives of IBD patients. ${ }^{162}$ Interestingly, epidemiological data using genomewide association studies indicate that a number of distinct genomic loci are involved in the genetic susceptibility to $\mathrm{CrD}$, including loci encoding genes involved in the maintenance of epithelial barrier integrity (reviewed in Van Limbergen et al. ${ }^{163}$ and in Xavier and Podolsky ${ }^{164}$ ). In addition, JAM- $A$ expression is downregulated in the inflamed colic epithelium in $\mathrm{CrD}$ and $U C,{ }^{30}$ and $J A M-A$-deficient mice, which exhibit decreased intestinal electrical resistance, increased fluorescein isothiocyanate-dextran-4000 permeability, and develop mucosal inflammation, ${ }^{31}$ indicating that a genetic defect in TJ structure might favor the induction of colitis. In addition, the absence of JAM-A in the intestinal epithelium is associated with increased susceptibility to dextran sulfate sodium (DSS) induced colitis, ${ }^{30}$ pointing to a role of JAM-A in preserving intestinal homeostasis. Another example is provided by SAMP1/YitFc mice for which the expression of TJ proteins is profoundly altered, although a defective gene remains to be identified. This defect is associated with the development of a spontaneous ileitis mimicking CrD. ${ }^{165}$ In these mice, impaired electrical resistance is observed independently of bacterial colonization, and several weeks before any detectable intestinal inflammation. These observations suggest that primary abnormalities in the expression of TJ proteins might favor the later development of IBD. The recent observation that genetic variants of the myo IXB gene are associated with IBD, ${ }^{166,167} \mathrm{CD},{ }^{168}$ and type I diabetes ${ }^{169}$ could suggest a primary defect in intestinal permeability in these diseases. However, contradictory results have been reported ${ }^{170}$ and a firm conclusion about the role of MYO IXB in the initiation of IBD cannot be drawn. Finally, it is notable that decreased electrical resistance, the downregulation of occludin as well as claudin-5 and -8 , and a strong upregulation of the pore-forming claudin-2 in active $\mathrm{IBD}^{171}$ contrast with the absence of any paracellular barrier dysfunction in patients with inactive $\mathrm{CrD}$, thus lending support against a primary defect. Taken together, no firm conclusions are available as yet regarding the primary role of intestinal permeability in the development of IBD.

\section{CONCLUSIONS}

Intestinal permeability is a generic term related to the absorption of various molecules ranging from small inert solutes (mannitol) to large ICs. In intestinal diseases, increased permeability to large molecules mostly (food antigens, microbial fragments) can have a deleterious role by exacerbating inappropriate immune responses. Irrespective of their transport pathway, paracellular or transcellular, it is mandatory to use adapted probes (proteins, bacteria) to delineate which material can cross the epithelial barrier. In that respect, small inert markers cannot mimic large molecules because of the size selectivity of the tight junctional pathway and an intestine leaky to small molecules can be tight to macromolecules. Obviously, no universal marker can be proposed which provides a definitive answer on the capacity of the intestinal mucosa to sense the intestinal content and to deliver antigens or bacteria to the underlying immune system. According to the extent of epithelial damage in diseases, increase 
in permeability can be related to the subtle opening of pores in the tight junctional complex, to an increased rate of transcytosis of antigens or ICs or at a final stage of inflammation, to apoptosis and ulceration leading to nonspecific leakage. It is important to keep in mind that beyond the controversies on paracellular vs. transcellular permeability, one important feature in intestinal diseases is the failure of the intestinal barrier to contain the macromolecular luminal content, a phenomenon likely to exacerbate unwanted immune responses.

\section{DISCLOSURE}

The authors declared no conflict of interest.

(C) 2010 Society for Mucosal Immunology

\section{REFERENCES}

1. Izcue, A., Coombes, J.L. \& Powrie, F. Regulatory T cells suppress systemic and mucosal immune activation to control intestinal inflammation. Immunol. Rev. 212, 256-271 (2006).

2. Strobel, S. \& Mowat, A.M. Immune responses to dietary antigens: oral tolerance. Immunol. Today 19, 173-181 (1998).

3. Macpherson, A.J., McCoy, K.D., Johansen, F.E. \& Brandtzaeg, P. The immune geography of IgA induction and function. Mucosal Immunol. 1, 11-22 (2008).

4. Cani, P.D. et al. Changes in gut microbiota control metabolic endotoxemia-induced inflammation in high-fat diet-induced obesity and diabetes in mice. Diabetes 57, 1470-1481 (2008).

5. Astwood, J.D., Leach, J.N. \& Fuchs, R.L. Stability of food allergens to digestion in vitro. Nat. Biotechnol. 14, 1269-1273 (1996).

6. Mahe, S., Messing, B., Thuillier, F. \& Tome, D. Digestion of bovine milk proteins in patients with a high jejunostomy. Am. J. Clin. Nutr. 54, 534-538 (1991).

7. Shan, L. et al. Structural basis for gluten intolerance in celiac sprue. Science 297, 2275-2279 (2002).

8. Shan, L. et al. Identification and analysis of multivalent proteolytically resistant peptides from gluten: implications for celiac sprue. J. Proteome. Res. 4, 1732-1741 (2005).

9. Diesner, S.C. et al. Dose-dependent food allergy induction against ovalbumin under acid-suppression: a murine food allergy model. Immunol. Lett. 121, 45-51 (2008).

10. Moreno, F.J. Gastrointestinal digestion of food allergens: effect on their allergenicity. Biomed. Pharmacother. 61, 50-60 (2007).

11. Travis, S. \& Menzies, I. Intestinal permeability: functional assessment and significance. Clin. Sci. (Lond) 82, 471-488 (1992).

12. Barker, N. et al. Identification of stem cells in small intestine and colon by marker gene Lgr5. Nature 449, 1003-1007 (2007).

13. Adson, A. et al. Quantitative approaches to delineate paracellular diffusion in cultured epithelial cell monolayers. J. Pharm. Sci. 83, 1529-1536 (1994).

14. Fihn, B.M., Sjoqvist, A. \& Jodal, M. Permeability of the rat small intestinal epithelium along the villus-crypt axis: effects of glucose transport. Gastroenterology 119, 1029-1036 (2000).

15. Powell, D.W. Barrier function of epithelia. Am. J. Physiol. 241, G275-G288 (1981).

16. Tavelin, S. et al. Prediction of the oral absorption of low-permeability drugs using small intestine-like 2/4/A1 cell monolayers. Pharm. Res. 20, 397-405 (2003).

17. Marcial, M.A., Carlson, S.L. \& Madara, J.L. Partitioning of paracellular conductance along the ileal crypt-villus axis: a hypothesis based on structural analysis with detailed consideration of tight junction structurefunction relationships. J. Membr. Biol. 80, 59-70 (1984).

18. Tsukita, S., Furuse, M. \& Itoh, M. Multifunctional strands in tight junctions. Nat. Rev. Mol. Cell Biol. 2, 285-293 (2001).

19. Furuse, M. et al. Occludin: a novel integral membrane protein localizing at tight junctions. J. Cell Biol. 123, 1777-1788 (1993).

20. Morita, K., Furuse, M., Fujimoto, K. \& Tsukita, S. Claudin multigene family encoding four-transmembrane domain protein components of tight junction strands. Proc. Natl Acad. Sci. USA 96, 511-516 (1999).
21. Mandell, K.J., McCall, I.C. \& Parkos, C.A. Involvement of the junctional adhesion molecule-1 (JAM1) homodimer interface in regulation of epithelial barrier function. J. Biol. Chem. 279, 16254-16262 (2004).

22. Ikenouchi, J., Furuse, M., Furuse, K., Sasaki, H. \& Tsukita, S. Tricellulin constitutes a novel barrier at tricellular contacts of epithelial cells. J. Cell Biol. 171, 939-945 (2005).

23. Furuse, M., Fujita, K., Hiiragi, T., Fujimoto, K. \& Tsukita, S. Claudin-1 and -2 : novel integral membrane proteins localizing at tight junctions with no sequence similarity to occludin. J. Cell Biol. 141, 1539-1550 (1998).

24. Lal-Nag, M. \& Morin, P.J. The claudins. Genome Biol. 10, 235 (2009).

25. Angelow, S. \& Yu, A.S. Claudins and paracellular transport: an update. Curr. Opin. Nephrol. Hypertens. 16, 459-464 (2007).

26. Colegio, O.R., Van Itallie, C.M., McCrea, H.J., Rahner, C. \& Anderson, J.M. Claudins create charge-selective channels in the paracellular pathway between epithelial cells. Am. J. Physiol. Cell Physiol. 283, C142-147 (2002).

27. Amasheh, S. et al. Claudin-2 expression induces cation-selective channels in tight junctions of epithelial cells. J. Cell Sci. 115, 4969-4976 (2002).

28. Krause, G. et al. Structure and function of extracellular claudin domains. Ann. NY Acad. Sci. 1165, 34-43 (2009).

29. Tamura, A. et al. Megaintestine in claudin-15-deficient mice. Gastroenterology 134, 523-534 (2008).

30. Vetrano, S. et al. Unique role of junctional adhesion molecule-a in maintaining mucosal homeostasis in inflammatory bowel disease. Gastroenterology 135, 173-184 (2008).

31. Laukoetter, M.G. et al. JAM-A regulates permeability and inflammation in the intestine in vivo. J. Exp. Med. 204, 3067-3076 (2007).

32. Hase, $\mathrm{K}$. et al. Uptake through glycoprotein 2 of $\mathrm{FimH}(+)$ bacteria by $\mathrm{M}$ cells initiates mucosal immune response. Nature 462, 226-230 (2009).

33. Kyd, J.M. \& Cripps, A.W. Functional differences between M cells and enterocytes in sampling luminal antigens. Vaccine 26, 6221-6224 (2008).

34. Worbs, T. et al. Oral tolerance originates in the intestinal immune system and relies on antigen carriage by dendritic cells. J. Exp. Med. 203, 519-527 (2006).

35. Kraus, T.A. et al. Induction of mucosal tolerance in Peyer's patch-deficient, ligated small bowel loops.. J. Clin. Invest. 115, 2234-2243 (2005).

36. Chirdo, F.G., Millington, O.R., Beacock-Sharp, H. \& Mowat, A.M. Immunomodulatory dendritic cells in intestinal lamina propria. Eur. J. Immunol. 35, 1831-1840 (2005).

37. Van Itallie, C.M. et al. The density of small tight junction pores varies among cell types and is increased by expression of claudin-2. J. Cell Sci. 121, 298-305 (2008).

38. Watson, C.J., Rowland, M. \& Warhurst, G. Functional modeling of tight junctions in intestinal cell monolayers using polyethylene glycol oligomers. Am. J. Physiol. Cell Physiol. 281, C388-C397 (2001).

39. Sugano, K. et al. Optimized conditions of bio-mimetic artificial membrane permeation assay. Int. J. Pharm. 228, 181-188 (2001).

40. Turner, J.R. Show me the pathway! Regulation of paracellular permeability by $\mathrm{Na}(+)$-glucose cotransport. Adv. Drug Deliv. Rev. 41, 265-281 (2000).

41. Bijlsma, P.B. et al. Differential in vivo and in vitro intestinal permeability to lactulose and mannitol in animals and humans: a hypothesis.

Gastroenterology 108, 687-696 (1995).

42. Fromm, M., Schulzke, J.D. \& Hegel, U. Epithelial and subepithelial contributions to transmural electrical resistance of intact rat jejunum, in vitro. Pflugers Arch. 405, 400-402 (1985).

43. Madara, J.L. \& Dharmsathaphorn, K. Occluding junction structurefunction relationships in a cultured epithelial monolayer. J. Cell Biol. 101, 2124-2133 (1985).

44. Weaver, L.T. \& Coombs, R.R. Does 'sugar' permeability reflect macromolecular absorption? A comparison of the gastro-intestinal uptake of lactulose and beta-lactoglobulin in the neonatal guinea pig. Int. Arch. Allergy Appl. Immunol. 85, 133-135 (1988).

45. Jalonen, T. et al. Increased beta-lactoglobulin absorption during rotavirus enteritis in infants: relationship to sugar permeability. Pediatr. Res. 30, 290-293 (1991).

46. Gotteland, M., Isolauri, E., Heyman, M., Tome, D. \& Desjeux, J.F. Antigen absorption in bacterial diarrhea: in vivo intestinal transport of betalactoglobulin in rabbits infected with the entero-adherent Escherichia coli strain RDEC-1. Pediatr. Res. 26, 237-240 (1989). 
47. Verdu, E.F. et al. The role of luminal factors in the recovery of gastric function and behavioral changes after chronic Helicobacter pylori infection. Am. J. Physiol. Gastrointest. Liver Physiol. 295, G664-670 (2008).

48. Neutra, M.R., Mantis, N.J. \& Kraehenbuhl, J.P. Collaboration of epithelial cells with organized mucosal lymphoid tissues. Nat. Immunol. 2, 1004-1009 (2001.).

49. Keita, A.V. et al. Characterization of antigen and bacterial transport in the follicle-associated epithelium of human ileum. Lab. Invest. 86, 504-516 (2006).

50. Keita, A.V. et al. Increased uptake of non-pathogenic E. coli via the follicle-associated epithelium in longstanding ileal Crohn's disease. J. Pathol. 215, 135-144 (2008).

51. Rescigno, M., Rotta, G., Valzasina, B. \& Ricciardi-Castagnoli, P. Dendritic cells shuttle microbes across gut epithelial monolayers. Immunobiology 204, 572-581 (2001).

52. Warshaw, A.L., Walker, W.A., Cornell, R. \& Isselbacher, K.J. Small intestinal permeability to macromolecules. Transmission of horseradish peroxidase into mesenteric lymph and portal blood. Lab. Invest. 25, 675-684 (1971).

53. Heyman, M., Ducroc, R., Desjeux, J.F. \& Morgat, J.L. Horseradish peroxidase transport across adult rabbit jejunum in vitro. Am. J. Physiol. 242, G558-564 (1982).

54. Gardner, M.L. Gastrointestinal absorption of intact proteins. Annu. Rev. Nutr. 8, 329-350 (1988).

55. Heyman, M., Grasset, E., Ducroc, R. \& Desjeux, J.F. Antigen absorption by the jejunal epithelium of children with cow's milk allergy. Pediatr. Res. 24, 197-202 (1988).

56. Wheeler, E.E., Challacombe, D.N., Kerry, P.J. \& Pearson, E.C. A morphological study of beta-lactoglobulin absorption by cultured explants of the human duodenal mucosa using immunocytochemical and cytochemical techniques. J. Pediatr. Gastroenterol. Nutr. 16, 157-164 (1993).

57. Heyman, M., Desjeux, J.F. \& Kaiserlian, D. Antigen handling by intestinal epithelial cells In Antigen Presentation by Intestinal Epithelial Cells (R.G.L. Company ed.) 1-16 (Springer, Heidelberg, 1996).

58. Terpend, K., Boisgerault, F., Blaton, M., Desjeux, J. \& Heyman, M. Protein transport and processing by human HT29-19A intestinal cells: effect of interferon gamma. Gut 42, 538-545 (1998).

59. Raposo, G. et al. B lymphocytes secrete antigen-presenting vesicles. J. Exp. Med. 183, 1161-1172 (1996).

60. Fevrier, B. \& Raposo, G. Exosomes: endosomal-derived vesicles shipping extracellular messages. Curr. Opin. Cell Biol. 16, 415-421 (2004).

61. Van Niel, G. et al. Intestinal epithelial cells secrete exosome-like vesicles. Gastroenterology 121, 337-349 (2001)

62. Mallegol, J. et al. T84-intestinal epithelial exosomes bear MHC class II/ peptide complexes potentiating antigen presentation by dendritic cells. Gastroenterology 132, 1866-1876 (2007).

63. van Niel, G. et al. Intestinal epithelial exosomes carry MHC class II/ peptides able to inform the immune system in mice. Gut 52, 1690-1697 (2003).

64. Coombes, J.L. et al. A functionally specialized population of mucosal CD103+ DCs induces Foxp3+ regulatory T cells via a TGF-beta and retinoic acid-dependent mechanism. J. Exp. Med. 204, 1757-1764 (2007).

65. Iliev, I.D. et al. Human intestinal epithelial cells promote the differentiation of tolerogenic dendritic cells. Gut 58, 1481-1489 (2009).

66. Almqvist, N., Lonnqvist, A., Hultkrantz, S., Rask, C. \& Telemo, E. Serumderived exosomes from antigen-fed mice prevent allergic sensitization in a model of allergic asthma. Immunology 125, 21-27 (2008).

67. Karlsson, M. et al. "Tolerosomes" are produced by intestinal epithelial cells. Eur. J. Immunol. 31, 2892-2900 (2001).

68. Fernandez, M.I. et al. Anti-inflammatory role for intracellular dimeric immunoglobulin a by neutralization of lipopolysaccharide in epithelial cells. Immunity 18, 739-749 (2003).

69. Lamm, M.E. Current concepts in mucosal immunity. IV. How epithelial transport of IgA antibodies relates to host defense. Am. J. Physiol. 274, G614-617 (1998)

70. Favre, L., Spertini, F. \& Corthesy, B. Secretory IgA possesses intrinsic modulatory properties stimulating mucosal and systemic immune responses. J. Immunol. 175, 2793-2800 (2005).
71. Mantis, N.J. et al. Selective adherence of IgA to murine Peyer's patch $M$ cells: evidence for a novel IgA receptor. J. Immunol. 169, 1844-1851 (2002).

72. Kadaoui, K.A. \& Corthesy, B. Secretory IgA mediates bacterial translocation to dendritic cells in mouse Peyer's patches with restriction to mucosal compartment. J. Immunol. 179, 7751-7757 (2007).

73. Weltzin, R. et al. Binding and transepithelial transport of immunoglobulins by intestinal $\mathrm{M}$ cells: demonstration using monoclonal IgA antibodies against enteric viral proteins. J. Cell Biol. 108, 1673-1685 (1989).

74. Boullier, S. et al. Secretory IgA-mediated neutralization of Shigella flexner prevents intestinal tissue destruction by down-regulating inflammatory circuits. J. Immunol. 183, 5879-5885 (2009).

75. Matysiak-Budnik, T. et al. Secretory IgA mediates retrotranscytosis of intact gliadin peptides via the transferrin receptor in celiac disease. J. Exp. Med. 205, 143-154 (2008).

76. Moura, I.C. et al. Identification of the transferrin receptor as a novel immunoglobulin (Ig)A1 receptor and its enhanced expression on mesangial cells in IgA nephropathy. J. Exp. Med. 194, 417-425 (2001).

77. Moura, I.C. et al. Glycosylation and size of IgA1 are essential for interaction with mesangial transferrin receptor in IgA nephropathy. J. Am. Soc. Nephrol. 15, 622-634 (2004).

78. Bonnefoy, J.Y., Aubry, J.P., Peronne, C., Wijdenes, J. \& Banchereau, J. Production and characterization of a monoclonal antibody specific for the human lymphocyte low affinity receptor for lgE: CD 23 is a low affinity receptor for IgE. J. Immunol. 138, 2970-2978 (1987).

79. Kaiserlian, D., Lachaux, A., Grosjean, I., Graber, P. \& Bonnefoy, J.Y. Intestinal epithelial cells express the CD23/Fc epsilon RII molecule: enhanced expression in enteropathies. Immunology 80, 90-95 (1993).

80. Negrao-Correa, D., Adams, L.S. \& Bell, R.G. Intestinal transport and catabolism of IgE: a major blood-independent pathway of lgE dissemination during a Trichinella spiralis infection of rats. J. Immunol. 157, 4037-4044 (1996).

81. Belut, D., Moneret-Vautrin, D.A., Nicolas, J.P. \& Grilliat, J.P. IgE levels in intestinal juice. Dig. Dis. Sci. 25, 323-332 (1980).

82. Yang, P.C., Berin, M.C., Yu, L.C., Conrad, D.H. \& Perdue, M.H. Enhanced intestinal transepithelial antigen transport in allergic rats is mediated by IgE and CD23 (FcepsilonRII). J. Clin. Invest. 106, 879-886 (2000).

83. Berin, M.C. et al. Rapid transepithelial antigen transport in rat jejunum: impact of sensitization and the hypersensitivity reaction. Gastroenterology 113, 856-864 (1997).

84. Bevilacqua, C. et al. Food allergens are protected from degradation during CD23-mediated transepithelial transport. Int. Arch. Allergy Immunol. 135, 108-116 (2004).

85. Yokota, A. et al. Two species of human Fc epsilon receptor II (Fc epsilon RII/CD23): tissue-specific and IL-4-specific regulation of gene expression. Cell 55, 611-618 (1988).

86. Montagnac, G. et al. Differential role for CD23 splice forms in apical to basolateral transcytosis of lgE/allergen complexes. Traffic 6, 230-242 (2005).

87. Tu, Y. et al. CD23-mediated lgE transport across human intestinal epithelium: inhibition by blocking sites of translation or binding. Gastroenterology 129, 928-940 (2005).

88. Li, H. et al. Transcytosis of lgE-antigen complexes by CD23a in human intestinal epithelial cells and its role in food allergy. Gastroenterology 131, 47-58 (2006).

89. Gastinel, L.N., Simister, N.E. \& Bjorkman, P.J. Expression and crystallization of a soluble and functional form of an Fc receptor related to class I histocompatibility molecules. Proc. Natl Acad. Sci. USA 89, 638-642 (1992)

90. Simister, N.E. \& Mostov, K.E. An Fc receptor structurally related to MHC class I antigens. Nature 337, 184-187 (1989).

91. Brambell, F.W. The transmission of immunity from mother to young and the catabolism of immunoglobulins. Lancet 2, 1087-1093 (1966).

92. Jones, E.A. \& Waldmann, T.A. The mechanism of intestinal uptake and transcellular transport of $\lg$ G in the neonatal rat. Gut 12, 855-856 (1971).

93. Jones, E. A \& Waldmann, T.A. The mechanism of intestinal uptake and transcellular transport of IgG in the neonatal rat. J. Clin. Invest. 51, 2916-2927 (1972).

94. Rodewald, R. pH-dependent binding of immunoglobulins to intestinal cells of the neonatal rat. J. Cell. Biol. 71, 666-669 (1976). 
95. Simister, N.E. \& Rees, A.R. Isolation and characterization of an FC receptor from neonatal rat small intestine. Eur. J. Immunol. 15, 733-738 (1985).

96. Israel, E.J. et al. Expression of the neonatal FC receptor, FCRn, on human intestinal epithelial cells. Immunology 92, 69-74 (1997).

97. Shah, U. et al. Distribution of the IgG Fc receptor, FcRn, in the human fetal intestine. Pediatr. Res. 53, 295-301 (2003).

98. Kim, J.K., Tsen, M.F., Ghetie, V. \& Ward, E.S. Localization of the site of the murine lgG1 molecule that is involved in binding to the murine intestinal Fc receptor. Eur. J. Immunol. 24, 2429-2434 (1994).

99. Tesar, D.B., Tiangco, N.E. \& Bjorkman, P.J. Ligand valency affects transcytosis, recycling and intracellular trafficking mediated by the neonatal Fc receptor. Traffic 7, 1127-1142 (2006).

100. Yoshida, M. et al. Human neonatal Fc receptor mediates transport of lgG into luminal secretions for delivery of antigens to mucosal dendritic cells. Immunity 20, 769-783 (2004).

101. Caulfield, M.J. \& Shaffer, D. Immunoregulation by antigen/antibody complexes. I. Specific immunosuppression induced in vivo with immune complexes formed in antibody excess. J. Immunol. 138, 3680-3683 (1987).

102. Machiels, J.J. et al. Allergic bronchial asthma due to Dermatophagoides pteronyssinus hypersensitivity can be efficiently treated by inoculation of allergen-antibody complexes. J. Clin. Invest. 85, 1024-1035 (1990)

103. Yoshida, M. et al. Neonatal Fc receptor for lgG regulates mucosal immune responses to luminal bacteria. J. Clin. Invest. 116, 2142-2151 (2006).

104. Bry, L. \& Brenner, M.B. Critical role of T cell-dependent serum antibody, but not the gut-associated lymphoid tissue, for surviving acute mucosal infection with Citrobacter rodentium, an attaching and effacing pathogen. J. Immunol. 172, 433-441 (2004).

105. Simmons, C.P. et al. Central role for B lymphocytes and CD4+ T cells in immunity to infection by the attaching and effacing pathogen Citrobacter rodentium. Infect. Immun. 71, 5077-5086 (2003).

106. Kobayashi, K. et al. An FcRn-dependent role for anti-flagellin immunoglobulin $\mathrm{G}$ in pathogenesis of colitis in mice. Gastroenterology 137, 1746-1756 e1741 (2009).

107. MacDonald, T.T., Hutchings, P., Choy, M.Y., Murch, S. \& Cooke, A. Tumour necrosis factor-alpha and interferon-gamma production measured at the single cell level in normal and inflamed human intestine. Clin. Exp. Immunol. 81, 301-305 (1990).

108. Raddatz, D., Bockemuhl, M. \& Ramadori, G. Quantitative measurement of cytokine mRNA in inflammatory bowel disease: relation to clinical and endoscopic activity and outcome. Eur. J. Gastroenterol. Hepatol. 17, 547-557 (2005).

109. Stallmach, A. et al. Cytokine/chemokine transcript profiles reflect mucosal inflammation in Crohn's disease. Int. J. Colorectal Dis. 19, 308-315 (2004).

110. Schock, F. \& Perrimon, N. Molecular mechanisms of epithelial morphogenesis. Annu. Rev. Cell Dev. Biol. 18, 463-493 (2002).

111. Bruewer, M., Samarin, S. \& Nusrat, A. Inflammatory bowel disease and the apical junctional complex. Ann. NY Acad. Sci. 1072, 242-252 (2006).

112. Utech, M. et al. Mechanism of IFN-gamma-induced endocytosis of tight junction proteins: myosin II-dependent vacuolarization of the apical plasma membrane. Mol. Biol. Cell 16, 5040-5052 (2005).

113. Matsuda, M., Kubo, A., Furuse, M. \& Tsukita, S. A peculiar internalization of claudins, tight junction-specific adhesion molecules, during the intercellular movement of epithelial cells. J. Cell Sci. 117, 1247-1257 (2004).

114. Bojarski, C. et al. Permeability of human HT-29/B6 colonic epithelium as a function of apoptosis. J. Physiol. 535, 541-552 (2001).

115. Schulzke, J.D. et al. Disrupted barrier function through epithelial cell apoptosis. Ann. NY Acad. Sci. 1072, 288-299 (2006).

116. Begue, B. et al. Implication of TNF-related apoptosis-inducing ligand in inflammatory intestinal epithelial lesions. Gastroenterology 130, 1962-1974 (2006)

117. Mankertz, J. et al. Expression from the human occludin promoter is affected by tumor necrosis factor alpha and interferon gamma. J. Cell Sci. 113 (Part 11), 2085-2090 (2000).

118. Zolotarevsky, Y. et al. A membrane-permeant peptide that inhibits MLC kinase restores barrier function in in vitro models of intestinal disease. Gastroenterology 123, 163-172 (2002).
119. Turner, J.R. et al. Physiological regulation of epithelial tight junctions is associated with myosin light-chain phosphorylation. Am. J. Physiol. 273, C1378-1385 (1997).

120. Su, L. et al. Targeted epithelial tight junction dysfunction causes immune activation and contributes to development of experimental colitis. Gastroenterology 136, 551-563 (2009).

121. Al-Sadi, R., Ye, D., Dokladny, K. \& Ma, T.Y. Mechanism of IL-1betainduced increase in intestinal epithelial tight junction permeability. J. Immunol. 180, 5653-5661 (2008).

122. Nusrat, A., Turner, J.R. \& Madara, J.L. Molecular physiology and pathophysiology of tight junctions. IV. Regulation of tight junctions by extracellular stimuli: nutrients, cytokines, and immune cells. Am. J. Physiol. Gastrointest. Liver Physiol. 279, G851-G857 (2000).

123. Scott, K.G., Meddings, J.B., Kirk, D.R., Lees-Miller, S.P. \& Buret, A.G. Intestinal infection with Giardia spp. reduces epithelial barrier function in a myosin light chain kinase-dependent fashion. Gastroenterology 123, 1179-1190 (2002).

124. Turner, J.R. Molecular basis of epithelial barrier regulation: from basic mechanisms to clinical application. Am. J. Pathol. 169, 1901-1909 (2006).

125. Yuhan, R., Koutsouris, A., Savkovic, S.D. \& Hecht, G. Enteropathogenic Escherichia coli-induced myosin light chain phosphorylation alters intestinal epithelial permeability. Gastroenterology 113, 1873-1882 (1997).

126. Madara, J.L. \& Stafford, J. Interferon-gamma directly affects barrier function of cultured intestinal epithelial monolayers. J. Clin. Invest. 83, 724-727 (1989).

127. Bruewer, M. et al. Proinflammatory cytokines disrupt epithelial barrier function by apoptosis-independent mechanisms. J. Immunol. 171, 6164-6172 (2003).

128. Bruewer, M. et al. Interferon-gamma induces internalization of epithelial tight junction proteins via a macropinocytosis-like process. FASEB J. 19, 923-933 (2005)

129. Rodriguez, P., Heyman, M., Candalh, C., Blaton, M.A. \& Bouchaud, C. Tumour necrosis factor-alpha induces morphological and functional alterations of intestinal HT29 cl.19A cell monolayers. Cytokine 7, 441-448 (1995)

130. Schmitz, H. et al. Tumor necrosis factor-alpha (TNFalpha) regulates the epithelial barrier in the human intestinal cell line HT-29/B6. J. Cell Sci. 112 (Part 1), 137-146 (1999).

131. Benlounes, N. et al. The threshold for immune cell reactivity to milk antigens decreases in cow's milk allergy with intestinal symptoms. J. Allergy Clin. Immunol. 98, 781-789 (1996).

132. Wang, F. et al. IFN-gamma-induced TNFR2 expression is required for TNF-dependent intestinal epithelial barrier dysfunction. Gastroenterology 131, 1153-1163 (2006).

133. Wang, F. et al. Interferon-gamma and tumor necrosis factor-alpha synergize to induce intestinal epithelial barrier dysfunction by upregulating myosin light chain kinase expression. Am. J. Pathol. 166, 409-419 (2005)

134. Schmitz, H. et al. Altered tight junction structure contributes to the impaired epithelial barrier function in ulcerative colitis. Gastroenterology 116, 301-309 (1999).

135. Heller, F. et al. Interleukin-13 is the key effector Th2 cytokine in ulcerative colitis that affects epithelial tight junctions, apoptosis, and cell restitution. Gastroenterology 129, 550-564 (2005).

136. Oshima, T., Miwa, H. \& Joh, T. Changes in the expression of claudins in active ulcerative colitis. J. Gastroenterol. Hepatol. 23 (Suppl 2), S146-150 (2008)

137. Kinugasa, T., Sakaguchi, T., Gu, X. \& Reinecker, H.C. Claudins regulate the intestinal barrier in response to immune mediators. Gastroenterology 118, 1001-1011 (2000)

138. Saunders, P.R., Kosecka, U., McKay, D.M. \& Perdue, M.H. Acute stressors stimulate ion secretion and increase epithelial permeability in rat intestine. Am. J. Physiol. 267, G794-799 (1994).

139. Gareau, M.G., Silva, M.A. \& Perdue, M.H. Pathophysiological mechanisms of stress-induced intestinal damage. Curr. Mol. Med. 8, 274-281 (2008)

140. Meddings, J.B. \& Swain, M.G. Environmental stress-induced gastrointestinal permeability is mediated by endogenous glucocorticoids in the rat. Gastroenterology 119, 1019-1028 (2000). 
141. Kiliaan, A.J. et al. Stress stimulates transepithelial macromolecular uptake in rat jejunum. Am. J. Physiol. 275, G1037-1044 (1998).

142. Santos, J. et al. Corticotropin-releasing hormone mimics stress-induced colonic epithelial pathophysiology in the rat. Am. J. Physiol. 277, G391-399 (1999).

143. Saunders, P.R., Hanssen, N.P. \& Perdue, M.H. Cholinergic nerves mediate stress-induced intestinal transport abnormalities in WistarKyoto rats. Am. J. Physiol. 273, G486-490 (1997).

144. Neunlist, M. et al. Human ENS regulates the intestinal epithelial barrier permeability and a tight junction-associated protein ZO-1 via VIPergic pathways 1. Am. J. Physiol. Gastrointest. Liver Physiol. 285, G1028-G1036 (2003).

145. Tripathi, A. et al. Identification of human zonulin, a physiological modulator of tight junctions, as prehaptoglobin-2. Proc. Natl Acad. Sci. USA 106, 16799-16804 (2009).

146. Fasano, A. et al. Zonulin, a newly discovered modulator of intestinal permeability, and its expression in coeliac disease 1. Lancet 355, 1518-1519 (2000).

147. Salama, N.N., Fasano, A., Lu, R. \& Eddington, N.D. Effect of the biologically active fragment of zonula occludens toxin, delta $\mathrm{G}$, on the intestinal paracellular transport and oral absorption of mannitol 1. Int. J. Pharm. 251, 113-121 (2003).

148. Drago, S. et al. Gliadin, zonulin and gut permeability: effects on celiac and non-celiac intestinal mucosa and intestinal cell lines 1. Scand. J. Gastroenterol. 41, 408-419 (2006).

149. Wang, W., Uzzau, S., Goldblum, S.E. \& Fasano, A. Human zonulin, a potential modulator of intestinal tight junctions 1. J. Cell Sci. 113 (Part 24), 4435-4440 (2000).

150. Schumann, M. et al. Mechanisms of epithelial translocation of the \{alpha\}2-gliadin-33mer in celiac sprue. Gut 57, 747-754 (2008).

151. Söderholm, J.D. et al. Epithelial permeability to proteins in the noninflamed ileum of Crohn's disease? Gastroenterology 117, 65-72 (1999).

152. Söderholm, J.D. et al. Increased epithelial uptake of protein antigens in the ileum of Crohn's disease mediated by tumour necrosis factor alpha. Gut 53, 1817-1824 (2004).

153. Schurmann, G. et al. Transepithelial transport processes at the intestinal mucosa in inflammatory bowel disease. Int. J. Colorectal Dis. 14, 41-46 (1999).

154. Kersting, S. et al. Antigen transport and cytoskeletal characteristics of a distinct enterocyte population in inflammatory bowel diseases. Am. J. Pathol. 165, 425-437 (2004).

155. Heyman, M. Gut barrier dysfunction in food allergy. Eur. J. Gastroenterol. Hepatol. 17, 1279-1285 (2005).
156. Stene, L.C. et al. Rotavirus infection frequency and risk of celiac disease autoimmunity in early childhood: a longitudinal study. Am. J. Gastroenterol. 101, 2333-2340 (2006).

157. Matysiak-Budnik, T. et al. Alterations of the intestinal transport and processing of gliadin peptides in celiac disease. Gastroenterology 125, 696-707 (2003).

158. Barisani, D. et al. Adaptive changes of duodenal iron transport proteins in celiac disease. Physiol. Genomics 17, 316-325 (2004).

159. Smecuol, E. et al. Gynaecological and obstetric disorders in coeliac disease: frequent clinical onset during pregnancy or the puerperium. Eur. J. Gastroenterol. Hepatol. 8, 63-89 (1996).

160. Ciacci, C. et al. Gender and clinical presentation in adult celiac disease Scand. J. Gastroenterol. 30, 1077-1081 (1995).

161. Boshuizen, J.A. et al. Changes in small intestinal homeostasis, morphology, and gene expression during rotavirus infection of infant mice 1. J. Virol. 77, 13005-13016 (2003).

162. Hollander, D. et al. Increased intestinal permeability in patients with Crohn's disease and their relatives. A possible etiologic factor. Ann. Intern. Med. 105, 883-885 (1986).

163. Van Limbergen, J., Wilson, D.C. \& Satsangi, J. The genetics of Crohn's disease. Annu. Rev. Genomics Hum. Genet. 10, 89-116 (2009).

164. Xavier, R.J. \& Podolsky, D.K. Unravelling the pathogenesis of inflammatory bowel disease. Nature 448, 427-434 (2007).

165. Kosiewicz, M.M. et al. Th1-type responses mediate spontaneous ileitis in a novel murine model of Crohn's disease. J. Clin. Invest. 107, 695-702 (2001).

166. Latiano, A. et al. The association of MYO9B gene in Italian patients with inflammatory bowel diseases. Aliment. Pharmacol. Ther. 27, 241-248 (2008).

167. van Bodegraven, A.A. et al. Genetic variation in myosin IXB is associated with ulcerative colitis. Gastroenterology 131, 1768-1774 (2006).

168. Monsuur, A.J. et al. Myosin IXB variant increases the risk of celiac disease and points toward a primary intestinal barrier defect. Nat. Genet. 37, 1341-1344 (2005).

169. Santiago, J.L. et al. Association of MYO9B haplotype with type 1 diabetes. Hum. Immunol. 69, 112-115 (2008).

170. Hunt, K.A. et al. Lack of association of MYO9B genetic variants with coeliac disease in a British cohort. Gut 55, 969-972 (2006).

171. Zeissig, S. et al. Changes in expression and distribution of claudin 2, 5 and 8 lead to discontinuous tight junctions and barrier dysfunction in active Crohn's disease. Gut 56, 61-72 (2007). 